\title{
Balkanologie
}

Balkanologie Revue d'études pluridisciplinaires

Vol. I, n 2 | 1997

Volume I Numéro 2

\section{Du conflit à la normalisation et de la normalisation au conflit}

(Les débats dans la presse albanaise sur les relations entre la Grèce et l'Albanie entre 1992 et 1996)

\section{Artan Fuga}

\section{(2) OpenEdition}

\section{Journals}

Édition électronique

URL : http://journals.openedition.org/balkanologie/215

DOI : 10.4000/balkanologie.215

ISSN : 1965-0582

Éditeur

Association française d'études sur les Balkans (Afebalk)

Édition imprimée

Date de publication : 1 décembre 1997

ISSN : 1279-7952

\section{Référence électronique}

Artan Fuga, «Du conflit à la normalisation et de la normalisation au conflit », Balkanologie [En ligne],

Vol. I, n² 2 | 1997, mis en ligne le 02 juin 2008, consulté le 17 décembre 2020. URL : http://

journals.openedition.org/balkanologie/215; DOI : https://doi.org/10.4000/balkanologie.215

Ce document a été généré automatiquement le 17 décembre 2020.

(c) Tous droits réservés 


\title{
Du conflit à la normalisation et de la normalisation au conflit
}

\author{
(Les débats dans la presse albanaise sur les relations entre la Grèce et
} l'Albanie entre 1992 et 1996)

\section{Artan Fuga}

1 Les relations entre la Grèce et l'Albanie ont trouvé un écho retentissant dans la presse albanaise durant les années 1990, années parfois difficiles du processus de transition postcommuniste en Albanie. Ces relations, devenant objet de discussions politiques, ont contribué à une différenciation encore plus nette entre les forces politiques albanaises, traditionnellement divisées entre nationalistes, en principe antigrecs, et "européens", qui voient dans le renforcement des liens politiques entre la Grèce et l'Albanie un des facteurs les plus importants, d'une part, de la stabilisation dans les Balkans, et, d'autre part, de l'intégration de l'Albanie dans le processus de l'unification européenne.

2 Un large spectre de problèmes divers et de dossiers chauds existent entre l'Albanie et la Grèce qui ne cessent de se manifester durant les années 1990. Certains problèmes créent une situation conflictuelle.

\section{Les conflits}

Les forces nationalistes albanaises et le gouvernement lui-même ${ }^{1}$ craignent l'ouverture rapide, économique et culturelle, du sud de l'Albanie, ayant peur d'une éventuelle hellénisation graduelle de cette zone et d'un développement déchaîné de la grécophilie. Le problème des Tchams ${ }^{2}$ représente une autre source permanente de conflit entre la Grèce et l'Albanie ; il attire depuis toujours l'attention de l'opinion publique albanaise, d'autant plus que les Tchams représentent actuellement une communauté influente dans la société albanaise. Les dirigeants albanais demandent que le gouvernement grec reconnaisse le droit de rapatriement et le droit de propriété sur les possessions que leur a confisqué l'État grec. 
4 Le problème de l'Église autocéphale albanaise demeure en vigueur. La présence de la religion orthodoxe en Albanie, particulièrement dans le sud du pays, complique davantage la situation ${ }^{3}$. Pendant les périodes conflictuelles, cette présence gêne les forces nationalistes albanaises, qui y voient une preuve de l'influence culturelle grecque à travers la frontière entre les deux pays ; par contre, pendant les périodes d'apaisement des relations entre les deux États, l'opinion publique cherche à développer un autre mythe et interprète ce fait comme un facteur de cohésion, d'amitié et de dialogue. Il ne s'agit pas du tout d'un problème abstrait, parce que la gravité de la situation se traduit immédiatement par de nombreux problèmes concernant l'administration de l'Église autocéphale albanaise et la compréhension même de cette nature autocéphale ${ }^{4}$. La nomination du prêtre Yanoullatos, citoyen grec, en tant que chef de l'Église autocéphale albanaise est devenu un sujet de débats interminables au niveau politique et médiatique et a été incorporé dans l'univers même des conflits traditionnels déjà présents.

5 La géopolitique de la région est également très complexe et parfois incompréhensible. En Albanie, on ne manque pas d'avoir et de manifester une certaine crainte envers une éventuelle alliance entre Belgrade et Athènes, qui mènerait une politique de déstabilisation à l'égard de l'Albanie chaque fois que celle-ci réclame le respect des droits de l'Homme au Kosovo. Athènes exigerait la même chose à propos de la minorité grecque en Albanie : autonomie pour autonomie, séparation pour séparation...

6 Ainsi, la politique grecque est considérée par les Albanais comme un second front subreptice organisé contre eux par les cercles nationalistes grecs au sud, qui, souvent, exercent leur influence sur le gouvernement grec et revendiquent le Vorio - Épire. La partie grecque exige des droits plus importants pour sa minorité par rapport aux droits existant aujourd'hui, comme par exemple un système éducatif plus étendu en grec, dans les programmes scolaires, mais aussi une extension de l'enseignement en langue grecque partout où se trouvent des minoritaires et pas seulement dans quelques zones restreintes du sud de l'Albanie où vivent traditionnellement les Grecs d'Albanie.

7 Des problèmes de nature politique se greffent à ces difficultés liées à des problèmes structurels, comme s'ils étaient insuffisants pour détériorer gravement la vie des habitants de cette région. Il s'agit de la dynamique politique à l'intérieur de chaque pays qui parfois se déroule à des vitesses politiques différentes. Il arrive que le parti au pouvoir en Albanie ait des affinités idéologiques avec le parti d'opposition en Grèce, ou, inversement, le parti d'opposition en Albanie fasse partie du même camp idéologique que le parti au pouvoir en Grèce. Ainsi, parfois, le gouvernement de chaque pays se voit plus proche de l'opposition politique de l'état voisin, et, par conséquent, tient des positions distantes par rapport au gouvernement en place. De cette façon, l'aspect idéologico-politique des relations a influencé, en général en les exaspérant, les sentiments de méfiance entre les deux pays. Dans ce cadre là, il faut souligner que les socialistes grecs, depuis leur arrivée au pouvoir, ont soutenu les socialistes albanais, dans l'opposition jusqu'en 1997.

8 Il devient très difficile de distinguer les choses. Le gouvernement d'un pays soutient-il l'opposition du pays voisin, afin d'exercer une pression au niveau des relations entre les deux États, ou bien ne s'agit-il que d'une politique affectée par des dogmes idéologiques et d'une fraternité de système de pensée politique? La question reste sans réponse claire : est-ce l'idéologique qui utilise le national et l'étatique, ou bien est-ce le national 
qui se sert de l'idéologique comme d'un simple instrument? Peut-être les deux réponses sont elles simultanément valables.

\section{Les intérêts communs}

9 et la Grèce n'est que la moitié du travail indispensable pour présenter la nature de ces relations.

L'Albanie et la Grèce possèdent également un espace commun d'intérêts de coopération : il semble qu'une politique albanaise pro-turque modérée, dans la mesure où la Grèce ne se sente pas menacée par l'Albanie, peut être récompensée par une politique grecque pro-serbe, modérée, dans la mesure où celle-ci n'empêche pas une solution pour les Albanais du Kosovo. Le marché albanais est plein de marchandises grecques, la Grèce y ayant trouvé un nouveau débouché. Par exemple, les importations en provenance de Grèce, au premier semestre 1994, occupaient la seconde place dans la totalité des importations, se plaçant ainsi derrière celles de l'Italie, premier pays importateur. Les importations en provenance d'Italie représentaient 34,97\%, alors que celles en provenance de la Grèce représentaient 25,79 \% des importations albanaises ${ }^{5}$.

11

Environ 300000 émigrés albanais travaillent en Grèce. Il ont fait baisser le prix de la force de travail non qualifiée, et accomplissent les travaux les plus difficiles, ce qui arrange quelque peu les propriétaires grecs de couche moyenne. Ce fait démographique et social porte en lui, implicitement, des tendances contradictoires. D'une part, on pourrait justement dire qu'il s'agit d'un facteur de stabilité des relations entre les deux pays. Toute agressivité de la politique albanaise envers la Grèce voisine trouverait un rempart très puissant, du fait de la présence des émigrés albanais en Grèce et de leurs propres familles en Albanie, donc, d'une masse très importante de la population. D'autre part, une agressivité politique grecque envers l'Albanie, doit compter sur la présence importante des Albanais en Grèce, où partout, que ce soit sur les places centrales d'Athènes ou dans les zones rurales, on entend parler albanais. On ne sait vraiment pas ce que cette "invasion" des "faibles" peut entraîner aussi bien culturellement, qu'au niveau de l'identité nationale en Grèce.

En revanche, la partie albanaise se trouve devant des réalités nouvelles. On a bien conscience qu'actuellement la société albanaise ne peut pas assimiler économiquement et socialement le retour brusque des Albanais travaillant et vivant en Grèce et en Italie. Un tel événement aurait renversé en quelques jours n'importe quel gouvernement de droite ou de gauche. Ainsi, chaque fois que l'Albanie persiste dans ses prises de position, résistant aux demandes grecques, la Grèce la menace du retour forcé des émigrés. Tous ces individus n'ont pas le droit de vote en Grèce. Ils ne peuvent pas, pour l'instant, influencer la politique grecque, mais ils (eux-mêmes et leurs familles) peuvent renverser toute prévision électorale, de par leur vote lors des campagnes électorales en Albanie. Ainsi, la situation devient paradoxale. La Grèce se voit "envahie" par des Albanais, parfois troublée par cette vague d'immigration qui change tout dans le pays, mais, de l'autre côté, par cette invasion même, elle se trouve dans un état de confort politique, capable d'influencer la vie politique albanaise.

13 Analysant toutes ces circonstances structurelles et conjoncturelles, statiques et dynamiques, on pourrait conclure que les relations entre la Grèce et l'Albanie sont toujours fragiles, prêtes à s'améliorer ou se détériorer en fonction d'un ensemble de 
facteurs ou d'influences qui peuvent émaner de l'extérieur même de ces deux pays. On pourrait aggraver facilement ces relations, si l'on souhaitait l'émergence d'une crise quelconque dans la région. En revanche, on pourrait les entraîner sans beaucoup de difficultés dans une perspective de coopération et de stabilité, si l'on cherchait une paix stable et durable dans les Balkans. Ces relations sont complexes. Elles se caractérisent par une influence physique, professionnelle et sociale prépondérante des Albanais en Grèce, et par une puissance virtuelle, politique et économique de la Grèce en Albanie. Les Albanais exercent leur influence envers la Grèce en tant qu'individus, tandis que l'influence grecque sur l'Albanie se réalise dans un cadre étatique. Autrement dit, au niveau des individus, les Albanais sont actuellement plus influents que les Grecs, bien qu'embauchés par ceux-ci; au niveau de la politique officielle, l'État grec a plus d'opportunités d'influencer la politique de l'État albanais, plutôt que l'inverse.

Une chose est importante : toute situation de crise ne peut plus sortir du cadre imposé par les nouvelles réalités économiques, sociales et culturelles dans les deux pays. Selon notre point de vue, un scénario catastrophe, une crise qui aboutirait à un conflit armé durable entre les deux États, hormis une conflagration généralisée obéissant à une autre logique géo-stratégique, est impossible.

Chaque fois que la situation devient conflictuelle, des mécanismes inhibants entrent en jeu et empêchent l'aggravation extrême du conflit. Par ailleurs, chaque fois que la normalisation revient, les mécanismes incitant les conflits commencent à agir, à cause de la normalisation et d'une certaine libéralisation du contrôle sur les facteurs de conflit. La situation ressemble à un état flou, à un va-et-vient du conflit qui tend vers la normalisation et de la normalisation qui tend vers le conflit. Il s'agit, donc, d'une situation de stabilité qui prépare l'arrivée du conflit, et d'un conflit qui s'achemine douloureusement vers une normalisation future. En d'autres termes, le conflit annonce l'arrivée de la normalisation et la normalisation prévient l'arrivée du conflit.

\section{Débats médiatiques et politiques}

16 À part l'incident militaire survenu lors de la pénétration de la frontière albanaise par un groupe armé grec, qui tua quelques soldats et officiers dans une caserne albanaise, nous citerons trois événements qui ont été à l'origine d'un dialogue très rude entre la presse écrite albanaise et grecque d'une part, et entre les forces politiques albanaises d'autre part.

\section{La déclaration commune entre le Pasok et le parti socialiste albanais}

17 En juin 1993, alors que le combat politique s'était aggravé en Albanie à cause de l'affaire $\mathrm{Nano}^{6}$, une délégation du Parti socialiste albanais se rendit en visite en Grèce et signa une déclaration commune avec le Parti socialiste grec, le Pasok. Les idées principales de cette déclaration étaient considérées par la presse socialiste comme une contribution à la paix et à la bonne attente dans les Balkans, tandis que pour la presse progouvernementale, il s'agissait d'une trahison des intérêts nationaux et d'un service que les socialistes albanais rendaient à la Grèce en échange de son soutien moral et matériel. Certaines formulations, dans la déclaration, firent l'objet de vives discussions, comme par exemple, l'affirmation que « les conflits dans les Balkans doivent être, avant 
tout, résolus par les Balkaniques $»^{7}$. Pour les socialistes, cette phrase exprimait l'idée de la limitation du conflit, alors que pour la presse adverse cela signifiait que les Albanais restent faibles et isolés, sans soutien extrabalkanique, dans des Balkans dominés par les Serbes, les Slaves et les Grecs. Cette réserve fut adoptée par les autres groupes politiques opposés au Parti socialiste, étant donné que dans la presse socialiste on déclare « que n'importe quel type d'intervention militaire (...) représenterait le danger de l'extension du conflit $»^{8}$.

Une seconde formulation soumise à controverse dans la presse albanaise de l'époque fut l'affirmation selon laquelle on soulignait dans la déclaration « la conviction ferme sur la non modification des frontières dans les Balkans " ${ }^{9}$. Pour les socialistes, cette affirmation exprime leur respect à l'égard des principes de la construction d'une nouvelle Europe, alors que pour leurs opposants, il s'agit de l'abandon délibéré et définitif des droits du peuple albanais du Kosovo pour l'autodétermination jusqu'à l'indépendance, droit dont jouirent les autres peuples de l'ex-Yougoslavie et qui fut favorablement accueilli par l'opinion internationale.

Une troisième formulation qui causa de grands débats, fut celle adoptée par le journal socialiste $L a$ Voix du Peuple qui, en analysant la déclaration en question, affirma que bien que tous acceptent, en principe, que l'Albanie se trouve en Europe et fasse partie de celle-ci, «la politique officielle albanaise a, pratiquement, été déséquilibrée, sans fondement, ni orientation prioritaire claire $»^{10}$. Si la presse socialiste affirme que toute sorte de nationalisme albanais contre le chauvinisme grec vise à mettre l'Albanie sous la tutelle d'une grande puissance extra-européenne, la presse pro-gouvernementale affirme que les socialistes albanais souhaitent intégrer l'Europe sous le contrôle grec.

\section{Le conflit autour de l'expulsion du prêtre Maidonis d'Albanie}

En juillet 1993, les organes compétents albanais décidèrent d'expulser le prêtre grec Maidonis accusé d'activité non religieuse visant à la désintégration de l'État albanais, notamment au travers d'une propagande vorio-épirienne. Les médias grecs réagirent vivement contre cette décision. Le Premier ministre grec de l'époque, Mitsotaqis, fit une déclaration dans laquelle il soulignait que l'affaire de Vorio-Épire, attaché à l'Albanie en 1913, est réelle et que la Grèce continuerait d'exiger le respect des droits de sa minorité en Albanie, tout comme celle-ci exige le respect des droits des Albanais en Serbie. En réaction, la police et l'armée grecques entreprirent une opération "coup de balai", c'est-à-dire poursuivirent et chassèrent violemment des milliers d'émigrés albanais en Grèce, en les tabassant, les emprisonnant, en les dépouillant même, dans certains cas, de l'argent gagné avec tant de peine dans des travaux pénibles. On conseillait alors aux Albanais poursuivis de «rentrer en Albanie pour renverser le président Berisha, et [de] revenir ensuite en Grèce ».

21 À la déclaration de Mitsotaqis et au "coup de balai", succéda la déclaration du Premier ministre albanais, Aleksander Meksi, dans laquelle il expliquait la position albanaise dans cette affaire. Selon lui, il n'y a aucun pouce de terre grecque en Albanie, et, par ailleurs, l'année 1913 fut celle du morcellement des territoires albanais et de l'occupation grecque du sud de l'Albanie. La minorité grecque en Albanie jouit de tous ses droits culturels, politiques et administratifs prévus par les actes internationaux. L'affaire des Albanais du Kosovo n'a rien à voir avec celle de la minorité grecque en Albanie, car les premiers représentent deux millions d'habitants et vivent, 
traditionnellement, sur leurs propres terres : ils ne sont pas, en conséquence, venus d'ailleurs. Il soulignait également que l'Albanie respecterait le statut de l'Église orthodoxe autocéphale albanaise. Il n'omit pas de critiquer la politique grecque à l'égard de ses minorités: "à cette occasion, nous rappellerons que des minorités ethniques et religieuses existent en Grèce, qui, non seulement, ne sont pas reconnues, mais aussi sont soumises à une atmosphère d'intolérance et d'arbitraire de la part des autorités, faisant ainsi de la Grèce l'objet de critiques de la part des organismes internationaux $»^{11}$. En soutien au gouvernement albanais, la presse publia l'intervention du président Sali Berisha, lors d'une rencontre avec le haut commissaire de l'ONU pour les minorités, Monsieur Van Der Stael :

Cette minorité, dit-il en parlant de la minorité grecque en Albanie, possède ses propres partis politiques et organisations sociales et politiques. Elle dispose de sa propre presse, jouit de la garantie totale des libertés d'opinion, de croyance, de circulation vers son pays d'origine, elle a ses propres députés et élus locaux là où ils ont gagné grâce au vote libre. Les enfants de cette minorité s'instruisent dans leur langue maternelle dans plus de 70 écoles de différents niveaux. Ils sont employés dans toutes les structures de l'État albanais. Le parti au pouvoir a nommé des minoritaires à des postes de ministres, de membres de la Cour Constitutionnelle, d'ambassadeurs et à d'autres postes de la fonction publique à tous les niveaux. ${ }^{12}$

22 En répondant au président de la République, le haut commissaire de l'ONU affirmait qu'» au cours de ma visite en Albanie, j'ai rencontré les représentants de la minorité. Ceux ci ne m'ont fait part d'aucun problème inquiétant $»^{13}$.

23 La presse albanaise adopta, de nouveau, des attitudes très différentes à l'égard de l'aggravation des relations entre l'Albanie et la Grèce. La presse d'opposition soutint que ces problèmes devaient être résolus par le dialogue et de façon constructive, que les responsables de cette situation étaient les cercles grecs chauvins et que le gouvernement albanais, de son côté, était incapable de construire des relations amicales avec les pays voisins, en particulier la Grèce.

Cette attitude passive, écrit l'Alliance, le journal du parti libéral l'Alliance Démocratique, en parlant de la politique gouvernementale albanaise, ne fait que renforcer nos doutes sur le fait que l'on conduise intentionnellement l'Albanie vers un nouvel isolement, cette fois-ci, dit "des portes ouvertes". Mais, il apparaît que des acteurs précis de la politique albanaise le souhaitent, tout comme ils souhaitent orienter l'Albanie vers des chemins politiques conflictuels pour des motifs dont l'Alliance Démocratique ne souhaite pas parler publiquement, avant d'en avoir discuté avec le gouvernement albanais. L'Alliance Démocratique, en rappelant au gouvernement albanais son opposition catégorique à la participation de l'Albanie à la Conférence Islamique, ainsi que son désaveu d'une politique des "blocs" dans les Balkans, desquelles résultent les pertes historiques de l'Albanie qui sont les conséquences, d'une part, de cette politique néfaste et, d'autre part, de l'alliance aux forces extra-européenne, fait sonner l'alarme sur les conséquences des développements à venir. ${ }^{14}$

24 Ainsi, on rendait responsable du conflit les pressions "extra-européennes" sur la politique albanaise. Cependant, dans la presse pro-gouvernementale, tout comme dans la presse proche des milieux albanais du Kosovo, on soutenait l'idée selon laquelle la politique grecque contre l'Albanie ne s'est pas modifiée depuis le début du siècle; elle s'est en effet consolidée en tant que structure immuable. Ainsi, dans La République, le journal du parti républicain, on publia un article où l'on décrivait la politique traditionnelle grecque et ses tactiques particulières, comme par exemple : 
l'infiltration (tout comme pendant la guerre de Troie) du camp adverse. Ainsi, on trouve les pro-grecs aussi bien dans le Parti de l'alliance démocratique, que dans le Parti socialiste, à l'association Omonia, comme à l'association des minoritaires grecs en Albanie, etc., dans les journaux L'Alliance, La Voix du Peuple, Notre Temps, etc., ou encore dans La Renaissance Démocratique, Le Front de la Nation, La République (du moins jusqu'à récemment), qui demeurent nationaux dans le fond ${ }^{15}$.

La presse de l'opposition est, donc, considérée infiltrée par la politique et l'action grecques en Albanie.

\section{Le procès des «têtes » de la minorité}

Lors de l'été 1994, cinq des dirigeants de l'Omonia furent arrêtés. En septembre, ils furent accusés d'espionnage, après l'abandon, par les procureurs, de la première accusation de trahison envers la patrie. Une véritable bataille médiatique eut lieu entre les médias grecs et les médias albanais et entre la télévision albanaise et les journaux de l'opposition, ainsi qu'entre les différentes tendances de la même presse albanaise autour du procès et de l'acte d'accusation. Quel était le contenu de cette accusation? Les minoritaires grecs auraient dû être condamnés car, selon l'accusation du procureur, ils avaient fourni des fusils de chasse à des membres de la minorité grecque en Albanie, armes d'ailleurs achetées en Grèce avec l'argent que leur avaient offert les services secrets grecs ; ils avaient vérifié les origines familiales, sélectionné et envoyé étudier en Grèce dans des académies militaires des "garçons de la minorité fidèles au VorioÉpire »; ils avaient dévoilé des informations diverses sur l'emplacement de casernes militaires albanaises dans la zone peuplée de minoritaires (vérifiées par les témoignages et les révélations des accusés durant l'instruction); ils avaient rédigé et rendu public les listes des officiers albanais d'origine grecque limogés de leurs fonctions ; enfin, pour avoir reçu des aides financières de Grèce pour le financement de la campagne électorale d'Omonia.

Le procès devint particulièrement complexe car une bonne partie des témoins ne se présenta pas au tribunal, se trouvant à l'étranger, et parce que les accusés déclarèrent avoir été contraints de témoigner pendant l'instruction de l'affaire et avoir subi une très forte pression psychologique de la part des juges d'instruction. Des dizaines de journalistes grecs assistèrent au procès, certains d'entre eux eurent même des problèmes avec la police albanaise qui leur interdisait l'accès à la salle d'audiences. Cependant, les médias grecs, et notamment la télévision, transmirent des interviews d'hommes politiques albanais de l'opposition. La télévision albanaise, de son côté, transmit au journal télévisé un reportage présentant des images saisies de la télévision grecque où des dirigeants politiques albanais de l'opposition et, en particulier, de l'Alliance démocratique, dans leurs interviews, semblent vouloir rendre le gouvernement albanais responsable de cette situation. Le titre de ce reportage était "ceux qui culpabilisent l'Albanie». Selon le commentaire du journaliste du journal télévisé, cette attitude témoignait du manque de patriotisme de la part de l'opposition, conciliante envers la politique grecque qui, sans hésiter, renouvelle l'opération "coup de balai" pour chasser violemment des dizaines de milliers d'émigrés albanais. Un avion militaire grec a même survolé le territoire albanais et propagé des tracts suggérant aux Albanais de renverser Berisha. Des porte-parole du gouvernement grec ont déclaré, ce qui s'est effectivement déroulé plus tard, qu'une telle attitude entraînerait le blocus des 35 millions d'écus accordés par l'Union européenne à 
l'Albanie, au cas où le gouvernement albanais rendrait coupables les détenus en question.

Néanmoins, les hommes politiques accusés par la télévision albanaise d'avoir donné des interviews à la télévision grecque, attaquèrent à leur tour celle-ci, l'accusant de transmettre de fausses informations au public albanais, au travers de montages (manipulations d'images et phrases extraites de leur contexte). Ainsi, par exemple, l'un d'entre eux, Namik Dokle, président du groupe parlementaire du Parti socialiste, écrit dans la Voix du Peuple :

Ce n'est pas une télévision, mais une inquisition médiévale. Pourquoi ne vient-elle pas recueillir nos opinions? Pourquoi choisit-elle des phrases détachées de leur contexte pour les tourner comme bon lui semble dans la bouche de Vjollca Vokshi [speakerine principale du journal télévisé - A. F.] ? La télévision albanaise souffre d'un complexe d'infériorité et n'ose pas affronter les hommes politiques de l'opposition ${ }^{16}$.

Dokle s'est vu obligé d'expliquer dans le journal de son parti son opinion sur le procès des cinq dirigeants de la minorité grecque. Il ne s'est pas prononcé sur le procès en lui même. Selon lui, « chaque État a le droit de juger ses citoyens ». Par contre, il exprime des réserves sur le fait que le procès des cinq minoritaires ne doive pas être considéré comme un procès «étendu à toute la minorité », mais n'explicite pas la signification de l'expression "étendu à toute la minorité ». Pour lui, la transformation de l'affaire judiciaire en affaire politique entre la Grèce et l'Albanie, aurait dû être évitée.

À propos de la réaction de la Grèce à cette affaire, N. Dokle se contente de critiquer l'aviation militaire grecque pour avoir pénétré l'espace aérien albanais, ainsi que la chasse aux émigrés albanais de Grèce. Mais, par ailleurs, il condamne les accusations de la télévision albanaise formulées à son égard et aux autres hommes politiques. Il accuse le Parti démocratique au pouvoir de mener une politique incorrecte, et la télévision albanaise d'être incapable de découvrir ce qui se cache derrière le trafic de pétrole entre la Grèce et la Serbie via le territoire albanais. «Je voudrais savoir, écrit-il, qui organise le trafic de carburant de la Grèce vers la Serbie ? Que la télévision albanaise filme les citernes qui vont et viennent, et qu'elle interroge ensuite tous les ministres, les députés, le Premier ministre et le président. ${ }^{17}$ N. Dokle parle d'une "facture de 80 millions de drachmes que le gouvernement de Mitsotaqis a donné au parti de Berisha (selon les témoignages au procès des cinq minoritaires) » et qui, selon lui, explique pas mal de choses.

D'après Halil Lalaj, député du Parti socialiste, les attaques de la télévision albanaise contre les hommes politiques de l'opposition, ayant adopté une attitude différente de celle du gouvernement au sujet de la crise avec la Grèce, découlent d'une mentalité primitive selon laquelle, en politique, il faut parler d'une seule et même voix, celle du chef de l'État. Tous ceux qui expriment une opinion différente de la sienne sont considérés comme des non-patriotes, au service de telle ou telle puissance étrangère : « la mentalité primitive de bey, écrit-il, est très néfaste. Pour les dirigeants actuels, il n'y a qu'un seul parti, il est le soleil et la lune, les autres partis jouent le rôle de figurants. Il y a un chef d'État "auto suffisant" en opinion, par conséquent, il n'a pas besoin de l'opinion des autres $»^{18}$.

N. Dokle s'exprime également sur la façon dont s'est déroulé le procès :

Il semble qu'il y ait eu des interrogatoires en l'absence d'avocat (...). On dit qu'Omonia a reçu des financements de l'État grec. Or, Omonia est une association et la loi n'interdit pas les aides données aux associations. Par ailleurs, le Parti 
démocratique a reçu des sommes énormes de « la Démocratie nouvelle » qui l'a aidé à prendre le pouvoir (...). Apparemment, il a été établi des listes de militaires touchés par la réforme. Et ceci semble être un secret ! L'association Omonia est une association qui ne s'est pas créée pour rien (...). Rien ne l'empêche de faire des listes de chômeurs, d'officiers, de policiers, d'agronomes (...). On dit aussi que les accusés ont parfois rencontré un certain Zarbakoullas à Joanina. On en conclut tout de suite : espionnage. Dire que ces façons de penser sont erronées, cela ne suffit pas. Les juges, à la mentalité communiste, devraient prouver ce dont on a parlé lors de ces rencontres, leurs liens, les dommages que cela a causé, etc. ${ }^{19}$

\section{L'univers du va-et-vient}

33 œuvre. L'Albanie a besoin de marchandises provenant de Grèce et les commerçants et les industriels grecs sentent la nécessité de l'ouverture du marché albanais. Il paraitt évident que, pour la Grèce, une Albanie posant, parfois, problème est plus souhaitable qu'une Albanie radicalisée dans ses positions fermes envers la Grèce et ralliée du côté de la Turquie. Le gouvernement albanais, de son côté, craint toujours une réaction négative de la part de la Grèce au sein des institutions européennes qui pèserait lourdement sur la situation économique et sociale du pays. On avance vers le compromis et une période d'entente ne manque pas de se déclencher. Alors, les éléments producteurs de crise se mettent lentement en marche. substituent réciproquement. Les deux types de discours ont leurs propres logique, symbolique et mythe primaire de légitimation. En ce qui concerne ce dernier élément, on pourrait dire que le langage de paix et de coopération est fondé sur le mythe des origines sublimées des Albanais et des Grecs dans le passé : "nous sommes les descendants des plus vieux peuples des Balkans, les Grecs et les Illyriens ». Pendant les temps de crise, on abandonne cet orgueil des origines anciennes et le discours devenu plus prosaïque, se fonde sur les querelles territoriales du début de ce siècle, au moment où l'Albanie se constituait en pays indépendant.

Balkanologie, Vol. I, n² 2 | 1997 

changement de rapport entre les gouvernements respectifs et les immigrés albanais en Grèce et la minorité grecque en Albanie. Le conflit et l'aggravation des relations entre les deux pays provoquent un éventail de problèmes concernant ces deux groupes sociaux. En Grèce on effectue des "coups de balai" contre les Albanais travaillant dans ce pays, tandis qu'en Albanie on devient plus prudent et plus rigide envers les demandes des minoritaires grecs concernant l'extension de leurs droits et de leurs libertés. Par contre, en période de stabilisation des rapports entre les deux pays, les Albanais travaillant en Grèce écoutent avec un grand espoir le discours prometteur des autorités grecques concernant leur régularisation, tandis que les minoritaires grecs en Albanie peuvent arracher plus de droits et de libertés ${ }^{20}$.

c) De la situation des relations entre les deux pays, conflictuelles ou coopératives, dépendent beaucoup les relations entre les communautés religieuses en Albanie, surtout les relations entre le clergé orthodoxe et le clergé musulman. Bien que les croyants, eux mêmes, restent un peu à l'écart du débat dogmatique, le discours médiatisé et le langage des hauts dignitaires orthodoxes et musulmans se modifie selon les circonstances. Toute crise entre les deux pays s'accompagne d'un discours conflictuel au niveau religieux et social de la part des chefs des communautés religieuses. Cela découle des réalités politico-sociales en Albanie. L'église orthodoxes albanaise, bien qu'autocéphale, est dirigée depuis des années par un prêtre de nationalité grecque. Cela est considéré par l'aile nationaliste albanaise comme une ingérence dans la vie sociale et religieuse de l'Albanie et elle perçoit, en conséquence, l'église orthodoxe comme un instrument de la politique grecque en Albanie. En outre, le débat s'envenime davantage du fait que la droite albanaise, nationaliste, garde fidèlement ses marques religieuses provenant de l'islam et considère l'islam comme un facteur traditionnel de la conservation de l'élément albanais contre les menées nuisibles des Slaves et des Grecs, tous orthodoxes. 


\section{NOTES}

1. En Albanie postcommuniste, de décembre 1991 à mars 1992, le Parti socialiste, conversion du Parti communiste (le Parti du travail de l'Albanie), était au pouvoir. Après les élections du 31 mars 1992, jusqu'en juin 1997, c'est le Parti démocratique, créé en 1991, principale force anticommuniste, qui a dirigé le pays. Après la crise et le conflit armé de la première moitié de 1997 en Albanie, conséquence de la faillite massive des sociétés financières frauduleuses, on a organisé de nouvelles élections, à la suite desquelles le parti socialiste est revenu au pouvoir.

2. Les Tchams sont des personnes d'origine albanaise et d'appartenance religieuse musulmane, citoyens grecs pourchassées par les autorités grecques après la Deuxième Guerre mondiale sous le prétexte de collaboration de certains Tchams avec les nazis dans le cadre de la constitution de la Grande Albanie.

3. Les Albanais sont majoritairement musulmans (70\%), mais $20 \%$ d'entre eux sont orthodoxes et $10 \%$ catholiques.

4. Les nationalistes albanais et les représentants de la communauté musulmane soutiennent l'idée que la nature autocéphale, selon le règlement intérieur de l'Église approuvé au début des années 1920, exclut toute nomination de citoyens étrangers à la tête de l'Église. Or, d'après un amendement de ce même règlement, approuvé dans les années 1950, la nature autocéphale de l'Église n'est pas incompatible avec la nomination d'un étranger comme chef de l'Église. Mais, les nationalistes soutiennent que les modifications des années 1950 ne sont pas valables parce qu'elles ont été formulées sous la pression du pouvoir communiste et totalitaire qui voulait le rapprochement de l'Église albanaise avec l'Église orthodoxe russe.

5. La statistique, (5), Publication de l'Institut des Statistiques de l'Albanie, Tirana, juin 1994.

6. Leader des socialistes albanais accusé de corruption et mis en prison par le pouvoir de l'époque, actuellement premier ministre de l'Albanie.

7. "Nouvelle page dans les relations entre le PS albanais et le Pasok», La Voix du Peuple, 18/06/93.

8. Ibid.

9. Ibid.

10. Ibid.

11. «Déclaration du premier ministre de la République d'Albanie, Aleksander Meksi, à propos des attitudes récentes du gouvernement grec, telles qu'exprimées par le premier ministre Mitsotaqis ", La Renaissance Démocratique, 21/07/93.

12. «La minorité grecque en Albanie jouit d'un standard des plus élevés en ce qui concerne le traitement des minorités », La Renaissance Démocratique, 30/07/93.

13. Voir La Renaissance Démocratique, 30/07/93.

14. «Mémoire du Parti l'alliance démocratique », L'Alliance, 06/07/93.

15. Ferraj (Hysamedin), « La Grèce, comme l'Albanie », La République, 04/07/93.

16. Dokle (Namik), «Télévision ou inquisition? », La Voix du Peuple, 28/08/94.

17. Ibid.

18. Lalaj (Halil), « Parler à voix unique », La Voix du Peuple, 28/08/94.

19. Dokle (Namik), art.cit.

20. Les chefs de la minorité ont été mis en liberté par décision du président de la Cour de Cassation d'Albanie, qui a commué la décision du tribunal en une peine avec sursis. 


\section{RÉSUMÉS}

Les relations gréco-albanaises reposent essentiellement sur le problème des minorités extérieures grecques et albanaises. Au travers du traitement médiatique de trois exemples dans la presse albanaise, l'auteur montre que ces relations fluctuent, d'une manière permanente, sur une échelle allant de la normalisation au conflit.

Relations between Greece and Albania depend, primarily, on their respective minority outside their mother country. Through the analysis of three examples in the Albanian newspapers, the author shows that these relations always fluctuate between normalization and conflict.

\section{AUTEUR}

\section{ARTAN FUGA}

Professeur des Universités (Albanie) ; Docteur en sciences de communication, Université Paris-II (France). 